\title{
Using Physical Experiments as Oracles
}

\author{
Edwin J. Beggs \\ Dept. of Mathematics, Swansea University, Wales SA2 8PP
}

In my talk I will consider how a digital computer (a Turing machine for the sake of being definite) could communicate with the physical world. Turing himself gave a mythological name to an external source of information for a computer an oracle. We shall consider how a physical experiment can be used to function as an oracle for a computer - a physical oracle. Thought experiments can be constructed using various physical theories, and we will examine their properties when used as oracles. The fundamental ideas we have to introduce into oracles for this are the time taken to perform the experiment, and the possibility of error in the answer.

On the side of the Turing machine, we ask what such an augmented machine can compute. To do this we consider the established area of nonuniform complexity, and how physical oracles act as advice functions. We can also look at the occurrence of error in the physical experiment, and show how this can also be expressed in terms of nonuniform complexity classes.

The time taken to perform an experiment can be unpredictable. If we consider how to get round this by implementing timers on the experiments we see that there are unexpected consequences for our ability to measure physical quantities.

On the side of physical theory, we ask whether there are limits on how much (or rather how quickly) information can be read from an experiment. This translates into the question of the trade off between the accuracy of an experiment and the time it takes to perform. By considering various experiments with various degrees of 'realism' we can suggest a common form of lower bound on the time taken to obtain a required accuracy.

Finally we can put the ideas of nonuniform complexity together with our proposed lower bound on the time for an experiment to formulate a conjecture on the upper bound of what a Turing machine connected to a physical oracle can compute in polynomial time - the class BPP//log*.

Joint work with John Tucker and Jose Felix Costa. 